\title{
An APL system for interactive data analysis: Histograms, correlation, and regression
}

\author{
SELBY EVANS and LYNNE GILFILLAN \\ Texas Christian University, Fort Worth, Texas
}

\begin{abstract}
This report describes an APL system for file-oriented univariate analysis, correlation, and regression analysis. The correlation and regression processes permit analyses of an unlimited number of cases, if the cases are arranged in tables small enough to be processed one at a time in a workspace. Support functions are also given for a menu-based processing system designed to support a broad range of analyses. The functions offer features such as subset selection, data transformation, and exclusion of invalid items.
\end{abstract}

This report presents several support functions for fileoriented data analysis. These, along with functions given in previous reports, allow a programmer to set up a menudriven data-analysis system with a simple, standard request format applying to all processes. The system allows menu-based selection of data columns, tables, and data subsets, with provisions to document the selection in the resulting output.

This report also presents a basic correlation function designed to operate on a file containing multiple data tables. The correlation function can analyze an unlimited number of cases if each table is small enough to be processed in the workspace. The results of this function can be used for multiple regression, principal components analysis, and canonical correlation, as well as for correlation analysis. Processing functions to produce correlation and multiple regression analyses are included.

The functions in this report have been developed and tested as part of a system. The descriptions given here should be interpreted as applying when the functions are part of the system. The functions are designed to operate on files organized by the data-preparation functions, TBLE and LABL, described by Evans, Neideffer, and Gilfillan (1982). Such files would have the data arranged in tables with subjects as rows and variables as columns. Three labeling components would follow the data tables. The first would carry the file title, a text vector identifying the contents and used as a title on each unit of output. The second would carry a scale table giving the ranges for each column, to be used in setting scales for histograms and plots. The third component would carry a twodimensional array with the names of columns and tables in the file.

The presentation assumes a workspace initialized by PARAM (Evans, Neideffer, \& Gage, 1981) and carrying previously defined functions TO, END (Evans et al.,

The authors' mailing address is: Department of Psychology, Texas Christian University, Fort Worth, TX 76129.
1982), and others noted in connection with specific functions given in later sections.

\section{GENERAL SUPPORT FUNCTIONS FOR FILE-ORIENTED DATA ANALYSIS}

Two functions, TIE and XEQ, provide basic operations needed for file-oriented data analysis. APL code for these functions is given in Figure 1. TIE accesses the data file and defines variables needed by XEQ and other functions. XEQ presents the menu and interprets user instructions. A third function, BOP (Evans et al., 1982), handles output of analyzed results and documents the analytic procedures.

These functions communicate with each other by transfer variables that must be global to these functions. The transfer variables described in this report are CLN, ATBN, DC, CL, ALL, TAB, NVL, delta-NV, and delta-TFR.

These functions use the following previously presented functions: TY, INTR (Evans et al., 1982).

TIE

TIE supports the accessing of labeled files. TIE gets the name of the file, checks to see that the file is in proper form for data analysis, and uses the labeling components to set up global variables carrying information needed by other functions.

TIE gets the name of the file by calling the more general file-tying function, TY, incorporating the features offered by that function. Thus, TIE remembers the name of the file last used and returns to it if the user responds to the file-name request by hitting return. TIE checks the file to detect common user errors. If the file is empty, has not been labeled, or does not contain tables, TIE reports the problem and terminates. These checks are hardly necessary for the experienced user, but they help protect the programmer from requests to explain what is wrong with the programs. Note that the check for a labeled file 


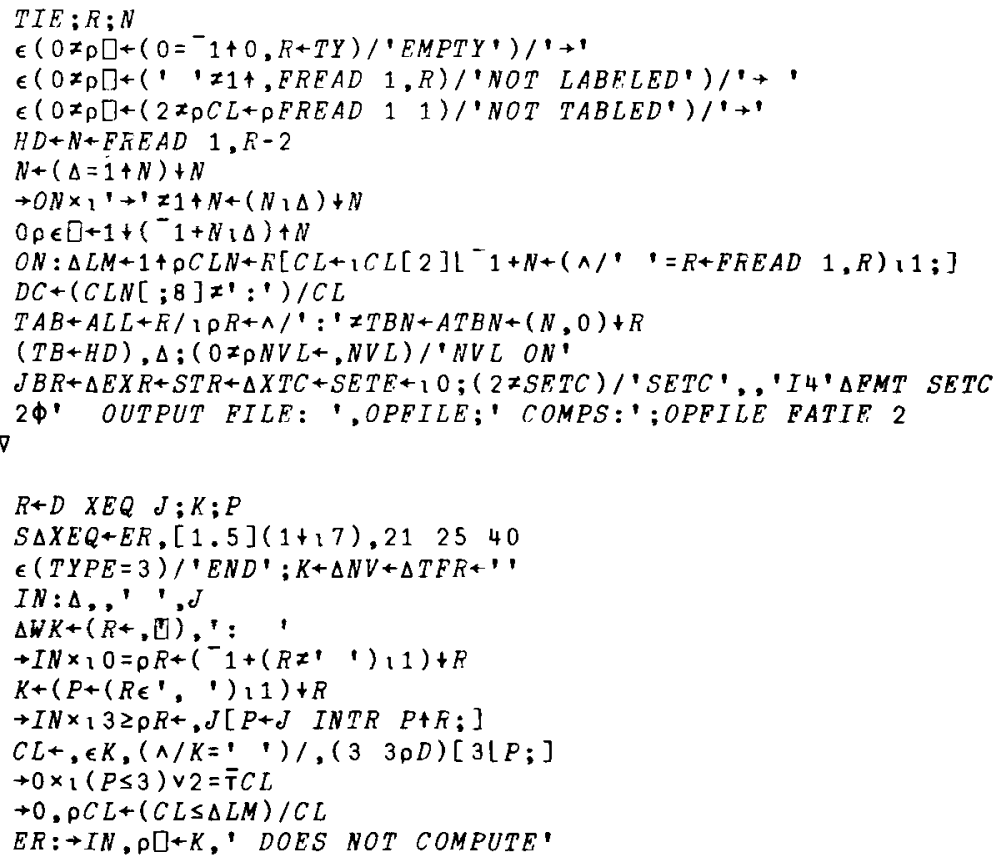

Figure 1. APL codes for general support functions TIE and XEQ.

is based on the assumption that the first character of the first column of the last component, which carries column and table names, is a blank. That condition will be met by files with labels formed by the previously defined function, LABL (Evans, Neideffer, \& Gage, 1980).

If the file is properly labeled, TIE uses the labeling components to create global variables and, otherwise, to arrange conditions for processing the file. It reads the array of column and file names in the last component and stores the column names in CLN and the table names in ATBN. TIE also forms a list of data column numbers on the basis of information in CLN. Columns carrying subject numbers and set-designating numbers are distinguished from columns carrying data to be analyzed by the presence of a colon as the last character of the column name. LABL automatically assigns a colon to columns 1 and 2 . The user with some APL skills can assign colons to other column names by hand.

The list of data column numbers is stored in the global variable DC and used by XEQ to let the user request analysis of all data columns without having to list them.

TIE also checks for the presence of the APL branching arrow (right pointing) as the first element in the second line of the title component. If the arrow is present, TIE applies the execute function to the remainder of that line. This feature permits the user to have certain commands executed each time the file is accessed. Typical uses would be to specify a particular name for the output file or to set the value of a control parameter. Associating a particular output file with a data file is convenient in that all processing results from a given file go to the same output file. If several data files are being analyzed in the same account, the results can be distributed to appropriate output files without user attention.

\section{$\mathbf{R} \leftarrow$ D XEQ J}

XEQ supports the function of menu-based processing functions. From the user's standpoint, XEQ presents a menu and accepts the user's request, correcting typographical errors and permitting the user to specify some subset of the total data file. If no subset is supplied by the user, XEQ supplies a default specification. For dataprocessing functions, the subset is defined by the columns to be analyzed. The default is the list of all data columns identified by TIE and stored in the global variable DC.

From the user's point of view, XEQ allows the use of a command such as REGQ 345 or REGQ 3 TO 6 to get multiple regression results based on the specified columns. Alternatively, the user can enter the command REGQ with nothing after to get multiple regression results for all data columns.

From the programmer's standpoint, XEQ handles the menu delivered in its right argument as an array with rows as menu items. XEQ presents this menu and accepts the user's request. To interpret the input, XEQ breaks the input into two parts. The part before the first blank is interpreted as a name in the menu, for example, REGQ. If there are any nonblank characters following the first blank, for example 3 TO 6, XEQ executes the string and stores the result in $\mathrm{CL}$; otherwise, the default column list is assigned to $C L$.

Input to $\mathrm{XEQ}$ is a semantic string with the menu function serving as the verb and the remainder treated as the object of the verb and stored in CL. In the example above, 
REGQ 3 TO 6 would be read by XEQ as a request to perform a linear regression using columns $3,4,5$, and 6 .

Note that XEQ does not actually invoke the processing function. With the aid of INTR, XEQ corrects minor typographical errors and delivers the name of the function as a text vector in the result, R. XEQ presents the menu again if it cannot recognize the user's entry. Execution is accomplished by the calling function, which applies the execute function to the result of XEQ.

Menu-based processing functions treat $\mathrm{CL}$ as the list of column numbers to be used in the analysis. If there is no explicit object, XEQ supplies a default value from the list given, as a character vector in the left argument. The character vector is first shaped as a $3 \times 3$ array. The default value for the first item on the menu is named in the first row of the array. The same correspondence applies to the second and third menu items. Menu items above the third get the same default as the third.

As given in Figure 1, XEQ is error trapped. Error trapping is not available on all systems, but is offered by I. P., by Sharpe time-sharing system, by Honeywell CP-V and CP-6 APL, by Univac's APL, by The Computer Company's APL68000, and by STSC's APL*Plus. The procedures vary somewhat from system to system, so we will describe the consequences of the error trap used in XEQ to aid in adapting the trap to other systems.

The effect of error trapping is to intercept APL's normal error-handling routines and insert an action selected by the programmer. The error trap is helpful for the novice user who might be confused by APL's usual error handling. If, for example, the user misspelled the name of a variable following a menu selection, the normal result would be an APL message indicating an undefined variable. The function would then be interrupted. In order to continue, the user would have to restart the process. With an error trap in place, this interruption can be avoided. The error trap, as used in XEQ, branches to an error message, such as "entry does not compute" and then presents the menu again, thus keeping the user within the system.

The trap is set on line 1. It leads to a trap on the following errors: undefined, error in syntax, domain, rank, length, or index. These are the errors most likely to result from user input error in this context.

For systems that do not support error trapping, lines 1 and 11 should be omitted. For systems that do support error trapping, it is probably best not to set the traps until the function is thoroughly tested. Debugging a function with an error trap is challenging, but not very rewarding.

\section{STANDARD MENU FUNCTIONS}

The menus presented by XEQ generally provide the user with a choice of two different types of functions: those that are dedicated to selection of data subsets; and those that actually initiate analytical procedures. The following three standard functions are used for selection of data subsets. The APL code for these functions is given in Figure 2.

\section{TABL}

From the user's point of view, typing in the menu selection TABL, followed by a numerical vector, results in the selection of the designated file components or tables of the data file for use in subsequent data analyses. For example, if TABL 157 were entered, then any dataanalysis functions subsequently selected, such as REGQ, would process only data found in components 1,5 , and 7. To return at any time to processing all tables, the user need only enter TABL with nothing following as a menu selection. The system automatically supplies the default list for all tables.

If the user invokes TABL to select data subsets, the names of the selected tables are put in the first line of each unit of output. The absence of any table list indicates that all tables were processed.

From the programmer's standpoint, TABL uses the transfer variable, $C L$, provided by $X E Q$, to identify the tables to be analyzed. As illustrated in the user interface function later in this report, TABL can be placed as the first item in the menu. XEQ permits a unique default value for that item, which would be named in the first three characters of XEQ's left argument. TIE stores a list of the component numbers of the data tables in the global variable ALL. Naming this variable in the appropriate place causes its contents to be delivered as the default value for $\mathrm{CL}$.

TABL stores the selected table numbers in TAB and the table names as an array in TBN. It also stores the table names as a raveled list in delta-TR. TAB and TBN are used by the processing functions to specify the tables to be processed and to identify them in the output. DeltaTR is used by BOP to provide the list of table names being processed in the output. Note that TIE specifies delta-TR as an empty vector, so that the output is not cluttered with table names unless table selection is in use.

\section{SETS}

For the user, SETS is a menu choice that permits the selection of a data subset for processing on the basis of values found in a specified column. For example, if the user types SETS 2 TO 5, the system responds by reporting the selected set numbers, in this case $2,3,4$, and 5 , and supplies the user with the name of the column most recently designated as a set column. It also offers the user an opportunity to change the number of the column designated for set selection. Then, in response to any subsequently selected processing functions, only those data rows are processed that have the specified set numbers, in this case $2,3,4$, or 5 , in the designated set column. To return to processing the entire data set, the user needs only to invoke the command SETS with nothing following. As was the case for TABL, set selection is reported in each unit of the output. 


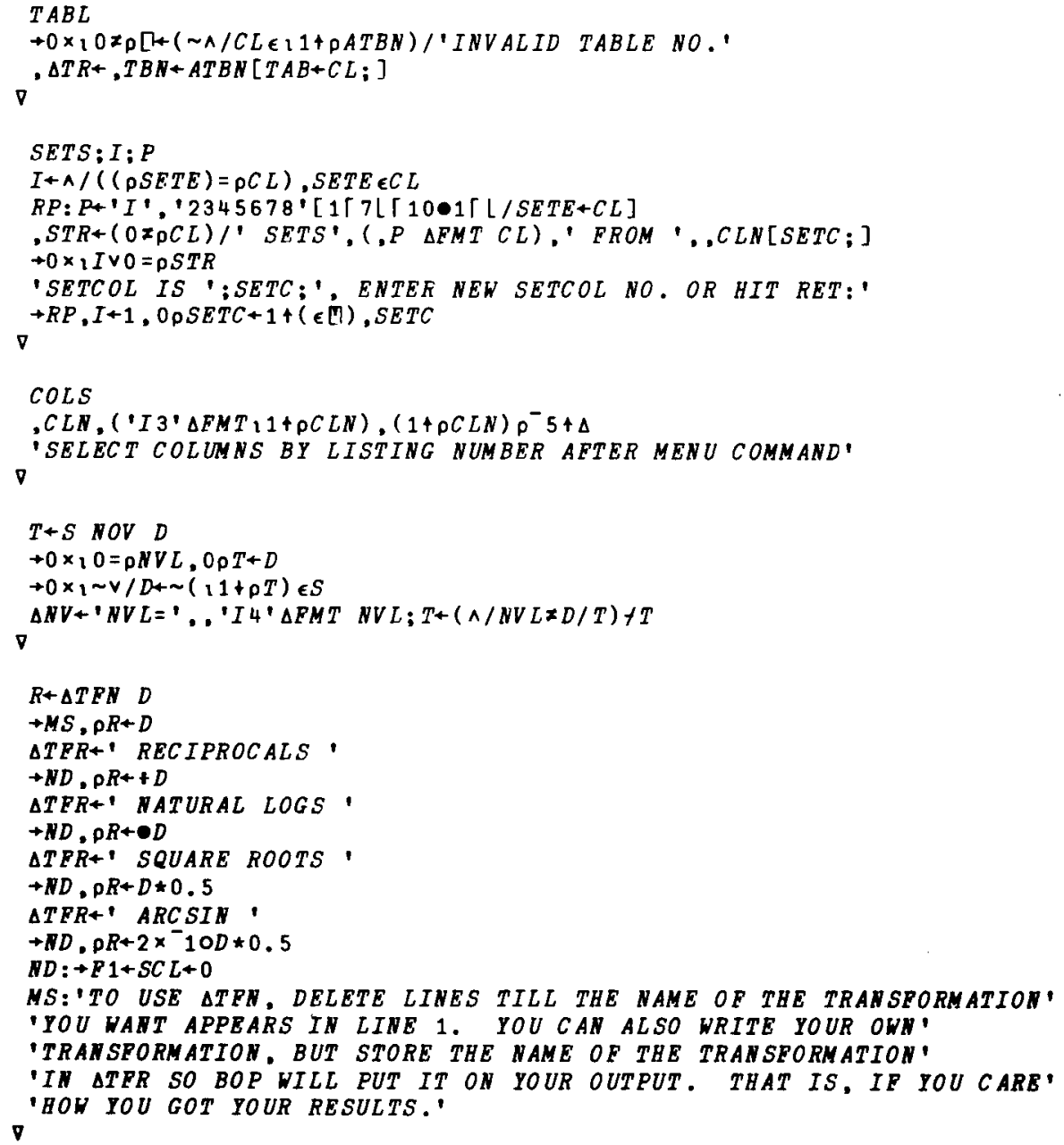

Figure 2. APL codes for menu functions TABL, SETS, and COLS, which are used for selection of data subsets, and for optional support functions NOV and delta-TFN.

From the programmer's standpoint, SETS is a standard menu function that activates set selection through SRD (Evans et al., 1981), referencing the specified set column and selecting items identified in the numerical vector following the command input. The command SETS, with nothing following, turns off set selection by supplying a default variable. SETS stores a report of the transaction in the transfer variable STR, to be used by BOP in identifying the output. When set selection is being turned off, no report is given and STR is set to the empty vector.

SETS uses the transfer variable, CL, provided by XEQ. Its handling is the same as that described for TABL.

\section{COLS}

COLS is provided as a user convenience and memory aid. When the user types COLS in response to a menu presentation, a list of column names and their related numbers is provided. This function assists in selecting column numbers for specifying data subsets.

\section{OPTIONAL SUPPORT FUNCTIONS}

The following functions, NOV and delta-TFN, provide for the exclusion of invalid data and for transformation of data before analysis. The functions are not presented by the menu and must be invoked by the user without benefit of a prompt. The functions are optional. They may be omitted from the system. If they are included, line 11 of BOP should be revised to include their transfer variables, delta-NV and delta-TFR, which carry reports of their operation to document the output. APL code for these functions is given in Figure 2.

\section{$T \leftarrow S$ NOV D}

From the user's point of view, NOV provides for selection of a data subset excluding data points having a user specified value, such as might be given to invalid items during data entry. The user invokes NOV by typing $N V L-X$, where $X$ can be any number. Then, in any subsequent analyses, the system will disregard any data row that carries the value $X$ in any data column. The user can turn NOV off by setting NVL to the empty vector. If NOV is used, the invalid data marker is specified in each unit of the output.

From the perspective of the programmer, NOV accepts a data table in its right argument, D, and delivers the same 
table as a result if the global variable NVL is an empty vector. If NVL carries a value, NOV strikes from the table any row with that value in a data column. Its left argument carries the column numbers to be treated as set columns, that is, to be disregarded in searching for invalid data points. If NOV operates, it leaves in the transfer variable delta-NV a report indicating that screening for invalid items was in operation and citing the value of the invalid data indicator.

NOV permits the user to work with tables containing invalid entries. It does not make maximum use of the data, since rows containing invalid entries are simply not processed. But NOV is simple and generally applicable to data-analysis programs. Assuming that the data table is reduced to the requested columns before being screened by NOV, a row with invalid entries will remain available for analysis if the columns containing invalid entries are not among those requested. This arrangement is included in the processing functions described later in this report.

\section{R $\leftarrow$ delta-TFN D}

Delta-TFN is an optional support function to aid in the investigation of nonlinear transformations of the data. It is given in two forms, a standard null form and an effective form. The standard form is kept in the main workspace. It has no effect on the results, but serves to let functions incorporate delta-TFN at the appropriate point, usually after the data table has been screened by eliminating invalid items, selection of subsets, and selection of data columns for analysis.

In its effective form, to be copied in from another workspace, delta-TFN allows the user to choose a transformation on the data and have it applied by processing functions. Instructions for the simplest use are carried in the function itself and are given to the user when the function is invoked. The operations in the function apply a selected transformation to all data columns. The user, however, can revise the function to process selected data columns.

\section{OPTIONAL FEATURES AND TRANSFER VARIABLES}

Features such as exclusion of invalid data and data transformations are optional for the programmer as well as for the user. The functions supporting these features can be omitted from processing functions without consequence for the rest of the processing. Conversely, the programmer can insert new features in the same way that these are inserted.

It is essential to recognize that the insertion of a feature is not complete until provision has been made for documenting the use of the feature in the output of results. Neglecting the documentation exposes the user to serious misinterpretation of the results.

The system given here permits the ready insertion of the documentation of new features by means of transfer variables that are generated when the process operates or when it is invoked. These variables transfer the report to BOP, which incorporates it into the output. Three steps are required for insertions.

First, arrange for the operation of the feature to store a report of the operation in a selected transfer variable. If the feature is invoked from the menu and is offered by all processing functions, the report may be generated by the menu function. This strategy is followed by TABL and SETS. If the feature is not invoked from the menu, or if it is not offered by all the processing functions, then the report should be generated by the function that provides the feature, as is done with NOV and delta-TFN. This arrangement ensures that the report is made only when the feature operates.

Second, insert the name of the transfer variable into line 11 of BOP.

Third, arrange to have the transfer variable set to the empty vector at the start of processing and again whenever the report becomes out of date. In the case of menuinvoked features, the report remains current until the feature setting is changed by the menu function or until the user interface function is terminated. Therefore, the transfer variables for these features are set to empty by TIE and set to the report values by their respective menu functions.

If not all the functions honor the feature, the report becomes outdated whenever a new function is invoked. Thus the report should be canceled by XEQ, which always precedes the execution of a function.

\section{BASIC FUNCTIONS FOR FILE-ORIENTED ANALYSIS}

The following functions provide file-oriented univariate statistics, correlation, and regression analyses. These functions refer to previously presented functions as follows: SUMR (Evans \& Gage, 1979), SRD (Evans et al., 1981), and FLAST (Evans, Neideffer, \& Gage, 1980). The first three functions presented, TVST, MREG, and SCLRD, are not presented as menu functions and are invisible to the user. Code for the functions is presented in Figure 3.

\section{$\mathbf{R} \leftarrow \mathbf{P}$ TVST $\mathbf{F}$}

TVST is a basic data analysis function that computes the correlation matrix and other commonly needed statistics for variables arranged as columns in one or more tables of a file. TVST allows the selection of columns and data subsets for processing and permits the exclusion of subjects (rows) with invalid data.

TVST operates on one table at a time, accumulating column sums and sums of squares and cross products in rawscore form. Since only one table is processed at a time, the number of subjects that can be processed is not limited by the workspace size. It is necessary only that each table be sufficiently small to be analyzed in a workspace. 


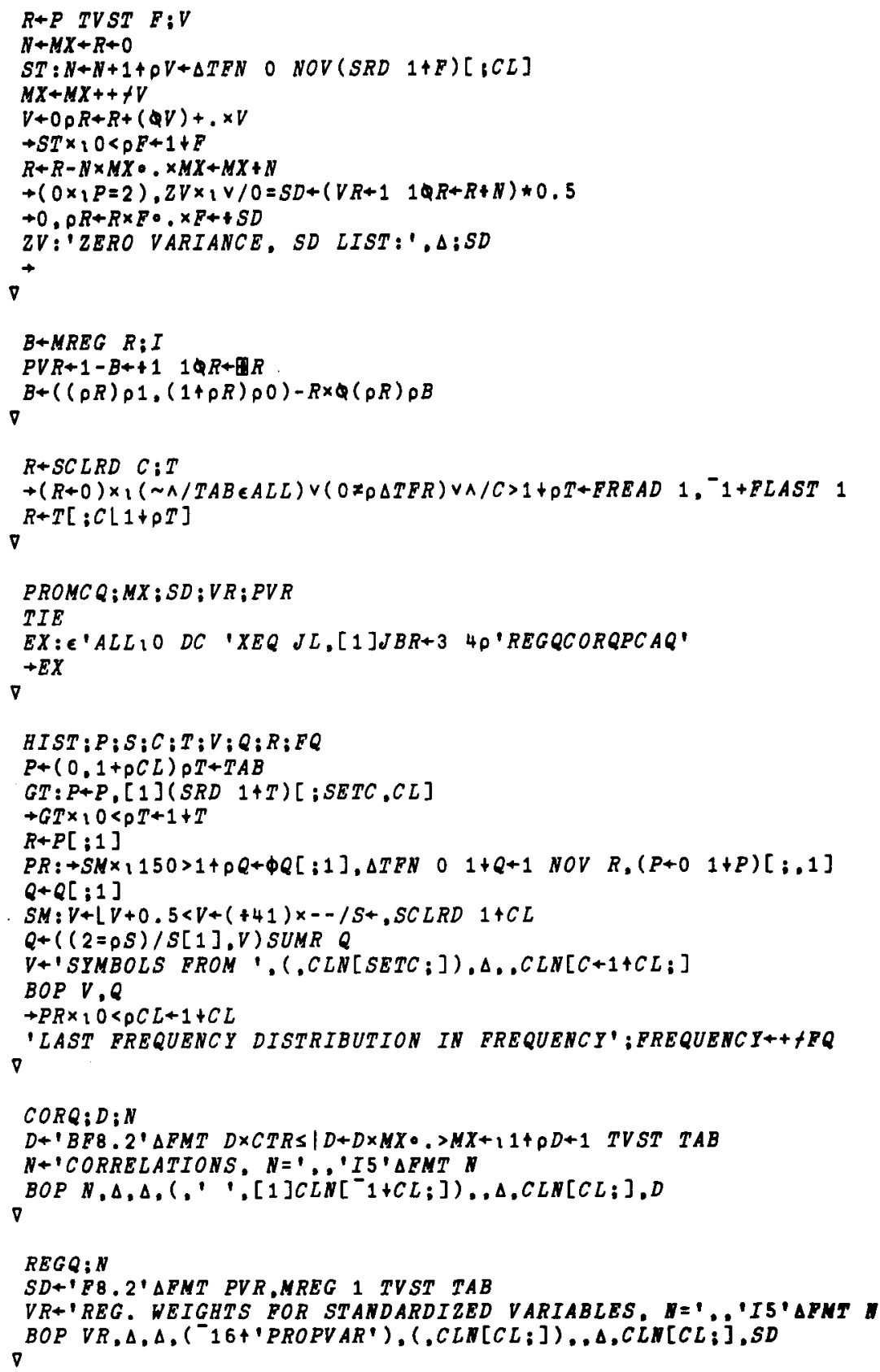

Figure 3. APL codes for basic functions TVST, MREG, SCLRD, PROMCQ, HST, CORQ, REGQ, which are used for file-oriented analyses.

TVST accepts in the right argument, $F$, a list of tables to be processed. The left argument is a control variable to select the result. For $P=1$, the result is the correlation matrix. For $P=2$, the result is the variancecovariance matrix. TVST also leaves the means, variances, standard deviations, and number of cases in the global variables MX, VR, SD, and N, respectively. The results are all ordered to correspond to the order of the data columns listed in the global variable, CL.

Since correlations cannot be defined for variables having zero variance, TVST detects and reports that con- dition. It gives the list of the standard deviations to aid the user in finding the problem and terminates the processing. This action avoids further processing of a meaningless correlation matrix. Experienced researchers will normally avoid this problem by inspecting the univariate statistics on their variables before going on to multivariate analyses.

\section{B - MREG R}

MREG is a basic multiple regression function that operates on the correlation matrix delivered in the right argu- 
ment to give normalized regression weights and proportion of variance accounted for. Instead of solving for a single criterion, it produces a regression solution for each variable in the correlation matrix.

This procedure for obtaining the solution is based on suggestions by Dubois and Manning (1960), who pointed out that the inverse of a correlation matrix contains all the information necessary to determine the multiple regression solutions for all of the variables simultaneously. Thus, one can get the solution for any desired criterion by including it with the predictor set in the correlation matrix.

The computations required for inverting the correlation matrix are very similar to those required for solving the simultaneous equations of a regression problem. Thus, the procedure adds little to the computation effort. It simplifies programming because it can be used with any existing function for getting a correlation matrix. Since it does not need to know which variable is intended as the criterion, the user input can be reduced to a listing of the variables to be used in the analysis. The additional information in the analysis of the predictors can also be useful, as discussed in connection with the illustration given in the next section.

In the result delivered in B, each row carries the normalized regression weights for one variable, in the same order as the variables appear in the correlation matrix. The columns of $B$ also correspond in order to the rows (or columns) of the correlation matrix and represent the variables to be weighted. The column position corresponding to the variable being predicted is set to zero. Thus, the first row of $B$, for example, could be used as a vector post multiplying the normalized data matrix to produce the predicted values of the first variable in the correlation matrix.

The proportion of variance accounted for by regression is delivered separately in the global variable PV, the order matching the order of columns in the correlation matrix.

If the correlation matrix is singular, MREG's attempt to find the inverse will result in an error, probably reported as DOMAIN ERROR or SING. MATRIX. The most likely sources of this problem are: One variable is the sum or average of other variables; the variables are required to have a fixed sum, as with proportions; or the number of cases is less than the number of variables. In general, one would want to have two or three times as many cases as variables and would discard variables, if necessary, to achieve this condition.

In virtually all cases, a singular matrix encountered by MREG implies that any other regression approach would also fail, although possibly in a different way. If the criterion were perfectly predictable from the predictors, a different procedure might succeed in cases in which MREG fails. Perfect prediction, however, would almost certainly be an indication that something is wrong with the data.

The algorithm used by MREG is not the optimal choice for accuracy. The high precision typical of APL calculations should be an adequate buffer against this problem. The algorithm might not be a suitable choice in other contexts.

\section{R - SCLRD C}

SCLRD is a support function designed to read the scale table from a labeled file and deliver ranges of values for the columns indicated in $\mathrm{C}$, making adjustments for columns not represented in the scale table. The scale table is formed by TSCALE (Evans, Neideffer, \& Gage, 1980), which limits the number of columns to 40 to avoid a possible workspace-full error when tables have too many columns.

If at least one of the requested columns is represented in the scale table, SCLRD substitutes the scale for the last column in the scale table for the missing scales. If none of the requested columns are represented in the scale table, SCLRD delivers zero as its result. As illustrated in this report, functions needing a scale are written to recognize this result as a request to fit the range to the available data.

As given here, SCLRD also delivers a zero if deltaTFR is not empty. This condition would indicate that delta-TFN has done a data transformation, making the original scale inappropriate. This test is effective only if SCLRD is invoked after delta-TFN has operated. Since delta-TFN is optional, this test is also optional and should be deleted in a system that omits delta-TFN.

The operation of SCLRD might seem so simple that it need not be written as a separate function. In the minisystem given here, the separation is not needed. But our system includes a number of plotting functions, some of which may be presented in subsequent reports. Separating the scale-reading process makes it simple to change the process without altering the functions that use it. Other programmers, for example, might want to notify the user when SCLRD uses a substitute scale, let the user enter a scale, or take some other action. These actions can be provided merely by using a modified version of SCLRD.

\section{PROMCQ}

PROMCQ is a user interface function designed to integrate the functions in this report into a single menu-driven system. It would be the only function directly invoked by the user. If the system were stored in its own workspace, the workspace would probably be set to start PROMCQ on loading. PROMCQ invokes TIE and XEQ. By applying the execute function to the result of XEQ, PROMCQ also invokes the functions requested by the user.

\section{HIST}

HIST is a processing function for menu use, providing univariate summary statistics for a data column in a labeled file. The results include the number of cases, the mean, the standard deviation, the $95 \%$ confidence interval, and a histogram. The user may enter HIST followed 
by a list of column numbers to be processed, or may enter only HIST and allow the system to use the default vector for specifying columns.

Programmers should note that the columns to be analyzed are delivered in the global variable $\mathrm{CL}$, defined by $\mathrm{XEQ}$. If more than one column is listed, HIST will process each column in turn. The results of HIST are produced by SUMR (Evans \& Gage, 1979) and its supporting functions.

\section{CORQ}

The user may enter CORQ in response to a menu presentation to generate a correlation matrix for the entire data set or for any subset selected by the functions SET and TABL. If CORQ is followed by a list of data column numbers, the user gets the correlation matrix for the data in the specified columns only. The results are presented as a labeled correlation matrix, including the number of valid cases processed.

From the programmer's point of view, CORQ is a processing function for menu use. It obtains the correlation matrix for the variables listed in the global variable CL over tables as specified by the global variable TAB. The results of CORQ are very similar in appearance to the results of CORRELATE (Evans, Gage, \& Neideffer, 1980). The simplification offered by the support of the system described here can be seen by comparing CORQ with CORRELATE.

\section{REGQ}

If the user selects REGQ from the menu, the system provides the normalized regression weights for each variable, in the order in which the variables are found in the data tables. Selection of data subsets is possible, using the same conventions described for HIST and CORQ.

To the programmer, REGQ is a processing function for menu use. It performs a multiple regression analysis on the variables listed in the global variable $C L$, over the tables listed in the global variable TAB. It delivers labeled results showing the proportion of variance accounted for and the regression weights for normalized variables. These results are given for each of the variables listed in $\mathrm{CL}$, in each case using the other variables listed in $\mathrm{CL}$ as the predictor set. The result is in the form of a table with rows representing separate solutions for each variable.

The results of an analysis with REGQ are given in Figure 4 . The data are variables 1,3 , and 4 of the array

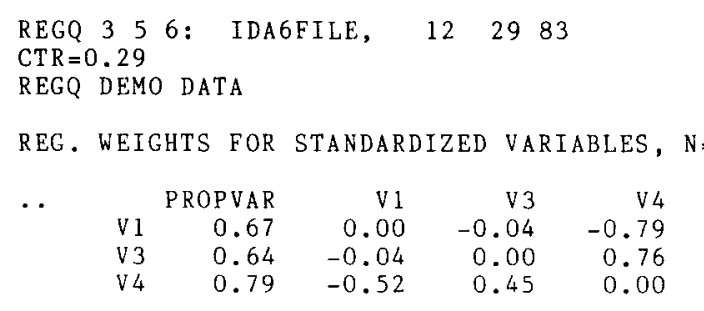

Figure 4. Results of regression analyses using REGQ. produced by CORGEN (Evans, Gage, \& Neideffer, 1980). The data set file is the same one used to illustrate PRINAN (Evans et al., 1981), except that the file was revised by splitting the data into two tables to illustrate the inclusion of multiple tables. The variables included in the regression were selected for illustrative purposes.

If Figure 4 represented real data with V4 as the intended criterion, the user might first note that $79 \%$ of the variance in V4 is accounted for by the other variables. Since the weights are for normalized variables, they are independent of the scale of measurement and can be meaningfully compared.

A glance at the other entries in the PROPVAR column shows that all the variables are well accounted for, indicating a great deal of shared variance. This observation suggests that not all of the variables would be required to achieve a high level of prediction for V4. On the basis of the weights, one might suspect that regression using only V1 or V3 would do a fairly good job. That speculation could be tested by a reanalysis with only one variable at a time, paired with V4. The result would show that each accounts for about $67 \%$ of the variance in V4.

By using data-selection options, the user can have REGQ carry out a judgmental stepwise regression by adding or deleting variables from the predictors. The judgmental basis for selection can incorporate factors such as the cost of measuring potential predictors, the suitability of the measure for replication in different laboratories, and the logical or scientific attractiveness of the measures. Although the weights give some indication of which variables should be retained, REGQ does not attempt to provide a good basis for conventional stepwise regression.

If used in this way, REGQ provides an alternative to stepwise regression for investigators who want to incorporate into the selection procedure background information not represented in the correlation matrix.

Any procedure that selects predictor variables by searching through a set of alternatives tested on the same data runs considerable risk of undesirable influence by chance characteristics of the data set. The comment applies both to conventional stepwise regression and to the less formal procedure described here. Problems with the replication of stepwise regression results have been noted (Wilkinson, 1979).

Tests of statistical significance are also potentially misleading in the context of interactive data analysis because they do not take into account the increased risk of Type I error introduced by selecting from alternative analyses. Inexperienced users may be lured into shopping for significant results. For that reason, REGQ does not give $\mathrm{F}$ ratios or statements of statistical significance.

The information needed to form an F or a t ratio is available in the results provided by MREG and TVST. Thus, REGQ could readily be modified to provide such information as might be appropriate for use in a noninteractive context.

A high level of shared variance may be favorable or unfavorable, depending on one's objectives. For determining a predictive equation, the condition may be quite 
favorable if the criterion participates in the sharing. In that case, the investigator may have a good range of options in selecting a final, reduced set of predictors.

For interpreting the weights, however, the existence of shared variance is unfavorable. Logically, variance that is shared by several predictors cannot be unequivocally ascribed to any of them. Technically, the confidence interval on the weights expands so much that the weights cannot be meaningfully compared.

The preceding discussion has dealt with the output of MREG for illustrative purposes. When viewed in the context of interactive data anlysis, however, the discussion is rather artificial. In practice, the result in Figure 4 would not have been obtained without prior analyses. A researcher would probably have started with HIST, inspect the distribution in each variable for skewness, bimodality, or other anomalies that would suggest that a variable was not well suited for use in multiple regression analysis.

After examining the univariate properties of the data, the researcher would probably inspect the correlation matrix (see Evans, Gage, \& Neideffer, 1980), noting a correlation of -.82 between V4 and V2 and a correlation of .82 between V4 and V3. That observation would be sufficient to identify $\mathrm{V} 2$ and $\mathrm{V} 3$ as promising predictors for V4.

\section{SUMMARY}

The support functions given here, along with those in previous reports, provide all of the elements needed to operate a menu-driven data-analysis system. The system provides a standard procedure for accessing data files, for making and recording selections of data subsets, and for invoking processing functions.

The system readily accommodates the addition of other processing functions, providing all or most of the user interface requirements so that the processing function can concentrate on its processes. From the user's standpoint, there is only one set of procedures to learn, and any process can be applied to any labeled data file. From the programmer's standpoint, the system handles the user interface, file access, and output operations. Commonly needed parameters are available in standard global variables. Selection processes can be incorporated, as appropriate, into the function by standard input functions. Programmer efforts can thus be directed at the special requirements of the process being developed.

In effect, these functions permit the development of a customized data-analysis system. Because the system is designed to operate effectively in a small workspace, it is especially suited for use on microprocessors. ${ }^{1}$

\section{REFERENCES}

Dubois, P. H., \& MANNing, W. H. (1960). An analytically meaningful approach to matrix inversion. Educational and Psychological Measurement, 20, 705-713.

Evans, S., \& GAGE, F. H. (1979). APL programs for interactive data analysis: Basic statistics and histograms. Behavior Research Methods \& Instrumentation, 11, 605-606.

Evans, S., GaGe, F. H. , \& NeIDEFFER, J. D. (1980). APL programs for interactive data analysis: Correlation and data entry. Behavior Research Methods \& Instrumentation, 12, 372-375.

Evans, S., Neidefrer, J. D., \& GaGe, F. H. (1980). APL functions for interactive data analysis: Graphics and labels. Behavior Research Methods \& Instrumentation, 12, 541-545.

Evans, S., NeidefFer, J. D., \& GaGe, F. H. (1981). APL functions for interactive data analysis: Principal components analysis. Behavior Research Methods \& Instrumentation, 13, 657-666.

Evans, S., NeidefFer, J. D., \& Gllfillan, L. (1982). APL functions for interactive data analysis: preparation of data files. Behavior Research Methods \& Instrumentation, 14, 475-481.

WILKINSON, L. (1979). Tests of significance in stepwise regression. Psychological Bulletin, 86, 168-174.

\section{NOTE}

1. The functions mentioned in this report have been adapted for use on the IBM PC, with STSC's APL*PLUS/PC. Instructions for making this adaptation can be obtained from the first author.

(Manuscript received March 21, 1984; revision accepted for publication September 1, 1984.) 